\title{
Dynamic modeling of managed pressure drilling applying transient Godunov scheme
}

\author{
Angel J. Sánchez-Barra ${ }^{1}$ - Rubén Nicolás-López ${ }^{2}$ - Oscar C. Valdiviezo-Mijangos² ${ }^{2}$ \\ Abel Camacho-Galván ${ }^{1}$
}

Received: 18 August 2014/Accepted: 17 May 2015/Published online: 29 May 2015

(c) The Author(s) 2015. This article is published with open access at Springerlink.com

\begin{abstract}
Transient hydraulics always characterizes the circulating flow during managed pressure drilling. Therefore, the application of the Godunov scheme to oil-well drilling hydraulics is presented. The numerical model developed describes the treatment process of the initial and boundary conditions from the well geometry and true operational conditions. The well-known finite-volume method and Riemann problem are utilized for building the set of discrete equations. The account of Godunov's simulation describes the profiles of transient pressure and transient flow rate along the well. For attending the oil-field engineering concerns, the drilling parameters discussed are as follows: choke pressure, pumping pressure, bottom-hole pressure, and circulating flow rate. After the comparison between computed and well data, the results show a small difference of less than 7 and $1 \%$ for pumping and bottomhole pressures, respectively. The main engineering contribution of this work is the solution and application of the first-order Godunov scheme to analyze the transient hydraulics during actual oil-well drilling and also the analysis and interpretation of the pressure wave behavior traveling along the well. The Godunov scheme has high-potential engineering applications for modeling the transient drilling hydraulics, i.e., controlled flow, underbalanced drilling, and foam cementing, as well.
\end{abstract}

Oscar C. Valdiviezo-Mijangos

ovaldivi@imp.mx

1 Facultad de Ingeniería, Universidad Nacional Autónoma de México, Ciudad Universitaria, Coyoacán, 04510 Mexico, DF, Mexico

2 Instituto Mexicano del Petróleo, Eje Central Lázaro Cárdenas 152, Delegación Gustavo A. Madero, 07730 Mexico, DF, Mexico
Keywords Pressure drilling - Oil-well hydraulics . Godunov scheme $\cdot$ Transient pressure $\cdot$ Transient flow

\section{Introduction}

Transient phenomena are always presented during oil-well drilling, as an implicit result of changing the flow rate, pumping, and choke pressures while the fluid "mud" is circulating through the well. To describe the transient hydraulics, the mathematical model composed by mass and momentum equations is applied,

$\frac{\partial \mathbf{U}}{\partial t}+\frac{\partial \mathbf{F}}{\partial x}=\mathbf{S}$

$$
\begin{gathered}
\mathbf{U}=\left[\begin{array}{c}
\mu \\
Q_{\mathrm{m}}
\end{array}\right], \quad \mathbf{F}=\left[\begin{array}{c}
Q_{\mathrm{m}} \\
A p+\frac{Q_{\mathrm{m}}^{2}}{\mu}
\end{array}\right], \\
\mathbf{S}=\left[\begin{array}{c}
0 \\
-f_{D}|u| u+\rho g A
\end{array}\right],
\end{gathered}
$$

where $\mu=\rho A, Q_{\mathrm{m}}=\mu u=\rho A u$, and $f_{D}=(f / 2) \cdot \rho /(2 \rho A)^{2}$ indicates the friction parameter, the variables $p, u, \rho, g$, and $A$ denote pressure, velocity, density, gravity constant, and cross-sectional area, respectively. Notice that to handle different wall roughness and flow rates, Moody friction factor $f$ can be directly added to Godunov scheme and computed in one step applying explicit correlations of $f$ (Bilgesu and Koperna 1995). This is suggested because the average error between explicit approximations and the implicit Colebrook relation is up to $3 \%$ (Brkić 2011). Explicit relations have to be a function of Reynolds number and relative roughness. Without altering research goals, an average friction factor of 0.015 is used because the flow is turbulent and relative roughness is always less than 0.0004 in all wellbore sections. 
To close the above system, the relationship between mass and pressure based on the definition of mixture sound celerity equation $c_{\mathrm{m}}$ is used as

$c_{\mathrm{m}}=\frac{c}{\left(1+\rho \eta_{\operatorname{Ref}} p_{\operatorname{Ref}}^{1 / \theta} / p^{(1+\theta) / \theta}\right)^{1 / 2}}$,

where $c$ is the celerity of the liquid pressure wave, $\eta_{\text {Ref }}$ is the gas fraction, and $\theta$ is equal to 1 and 1.4 for isothermal and adiabatic conditions, respectively. The limiting case is for pure liquid flow with any presence of gas, $c_{\mathrm{m}}=c$.

After this brief explanation of the mathematical model, the initial and boundary conditions to describe the drilling hydraulics are itemized as

$\mathbf{U}(x, 0)$, well data in $0 \leq x \leq L$

$Q_{\mathrm{m}}(0, t), \quad$ constant flow rate

$p(L, t)$, choke pressures.

The first statement denotes the initial condition. It means that entire oil-well conditions are known at $t=0$, usually considering static or steady well data. The second represents constant liquid flow rate at the left boundary. The last one corresponds to the right boundary condition and it is closely related to managed pressure drilling. Both of them remove unnecessary complexity without sacrificing accuracy.

The discrete solution of the initial-boundary mathematical model applying the Godunov scheme is supported by a set of Riemann problems. All variables listed at $\mathbf{U}(x, t)$, Eq. 1 are evaluated at $x=x_{0}$ according to

$\mathbf{U}(x, t)=\left\{\begin{array}{ll}\mathbf{U}_{\mathrm{L}} & \text { for } x \leq x_{0}-c_{\mathrm{m}} t \\ \mathbf{U}^{*} & \text { for } x_{0}-c_{\mathrm{m}} t<x \leq x_{0}+c_{\mathrm{m}} t \\ \mathbf{U}_{\mathrm{R}} & \text { for } x>x_{0}+c_{\mathrm{m}} t\end{array}\right\}$,

where $\mathbf{U}_{\mathrm{L}}, \mathbf{U}^{*}$, and $\mathbf{U}_{\mathrm{R}}$ are the left, intermediate, and right states of the Riemann problem, respectively. These definitions are relevant during the time integration process, whereas the numerical fluxes are reconstructed. The final numerical model based on the finite-volume method is rigorously developed and solved using the definition of Riemann problem (Eq. 4) for the entire physical domain and time length of simulation.

Godunov scheme is a modern shock-capturing method and its main advantage is that there is no need to track interfaces or discontinuities explicitly. As a result of this advantage, Godunov scheme is applicable to problems involving smooth solutions, discontinuous solutions, and complex wave interaction. On the other hand, conventional numerical schemes need continuous solutions (i.e., finite difference method) and most of them were not designed to capture contact discontinuities, for instance, compressive or rarefaction shock. In previous works, the Godunov scheme has been recently applied to analyze transient twophase flow in rectangular and circular pipes for different research purposes (Kerger et al. 2011; Bousso and Fuamba 2013). However, Godunov scheme is a useful numerical tool for dealing with free-surface gravity flow, compressible flow or multiphase flow. Technical literature is very rich on these topics.

Therefore, the main engineering contribution of this work is the solution and application of the first-order Godunov scheme to analyze the transient hydraulics during actual oil-well drilling, because this numerical scheme is easy to implement and encode in any programming language (Guinot 2001). Here, the chosen hydraulics of the field operation is defined as managed pressure drilling. To achieve it, how to implement the set of initial and boundary conditions taking in account the oil-well geometry and real operational conditions of the drilling hydraulics was discussed. The analysis and interpretation of the pressure wave behavior traveling along the well are also included.

Finally, the numerical results of the drilling simulation are discussed in detail for the most important parameters: choke pressure, pumping pressure, bottom-hole pressure, and circulating flow rate; these are widely validated through reported oil-well data in a standard of the American Petroleum Institute standards (API RP-13D 2003).

\section{Basis of the Godunov scheme}

The Godunov scheme is extensively used for modeling shock and contact discontinuities. Here, the steps of the well-documented first-order Godunov scheme (Toro 2009) are consistently applied to solve the complete set of the transient model described by Eqs. 1-4. We start defining local finite volumes or cells on the entire length of the physical domain. The discretization is carried out on $x$ axis
Fig. 1 Numerical cells for boundaries and internal interfaces of the computational domain

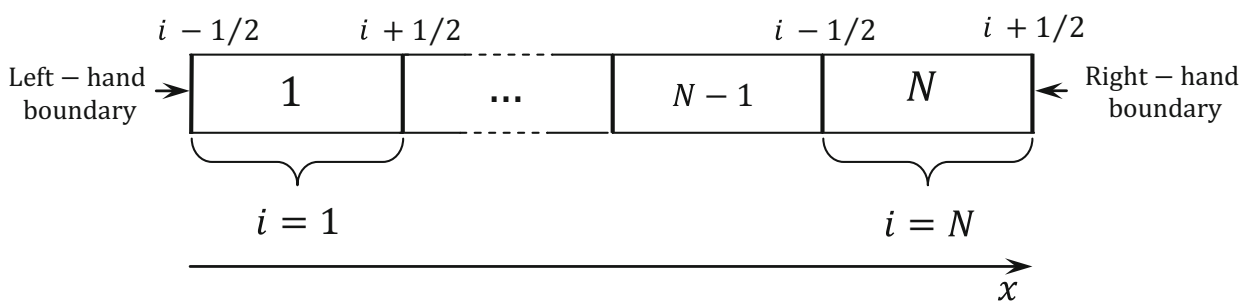


from $i=1$ to $i=N$ and on the time spacing from $t=n$ to $t=n+1$. Figure 1 illustrates that left-hand and righthand boundaries are located at $i=1 / 2$, and $i=N+1 / 2$, respectively. Additionally, the internal cells are indicated by $i=1$ to $i=N$.

The flux computation for the internal interfaces $\mathbf{F}_{i+1 / 2}^{n+1 / 2}$ is based on the next procedure

$\mathbf{F}_{i+1 / 2}^{n+1 / 2}=\mathbf{F}\left(\mathbf{U}_{i+1 / 2}^{n+1 / 2}\right)=\left[\begin{array}{c}Q_{\mathrm{m}, i+1 / 2}^{n+1 / 2} \\ A p_{i+1 / 2}^{n+1 / 2}\end{array}\right]$,

where $\mathbf{U}_{i+1 / 2}^{n+1 / 2}$ is the solution of the Riemann problems stated at Eq. 4 for the cell interfaces. Taking in account the definition of $Q_{\mathrm{m}}=\mu u$, the first component is computed as $Q_{\mathrm{m}, i+1 / 2}^{n+1 / 2}=\mu_{i+1 / 2}^{n+1 / 2} u_{i+1 / 2}^{n+1 / 2}$.

For each internal interface located at $i+1 / 2$, from $i=1$ to $i=N-1$, both $\mu_{i+1 / 2}^{n+1 / 2}$ and $u_{i+1 / 2}^{n+1 / 2}$ are calculated from the next equations

$$
\begin{aligned}
& \mu_{1 / 2}^{n+1 / 2}=\left[1+\frac{u_{b}-u_{1}^{n}}{c_{1}^{n}+c\left(\mu_{1 / 2}^{n+1 / 2}\right)}\right] \mu_{1}^{n} \\
& \mu_{N+1 / 2}^{n+1 / 2}=\left[1+\frac{u_{N}^{n}-u_{b}}{c_{N}^{n}+c\left(\mu_{1 / 2}^{n+1 / 2}\right)}\right] \mu_{1}^{n}
\end{aligned}
$$

After establishing the discrete equations of interface fluxes for internal and boundary cells, it is essential to assure numerical stability in all cells. Therefore, the next step is to define a computational time step less than the maximum time step $\Delta t_{\max }$

$\Delta t_{\max }=\underset{i=1, \ldots, N}{\operatorname{Min}}\left(\frac{\Delta x_{i}}{|u|+c_{\mathrm{m}}}\right)$.

It is valid since an analytical solution is applied to evaluate the source term.

Finally, the evolution of $\mathbf{U}(x, t)$ from $t=n$ to $t=n+1$ is assessed in two parts. The homogeneous pure advection part is solved by the balance over the time-space domain

$$
\left.\begin{array}{c}
\left(c_{i}^{n}+c_{i+1 / 2}^{n+1 / 2}\right)\left(\mu_{i+1 / 2}^{n+1 / 2}-\mu_{i}^{n}\right)+\left(\mu_{i}^{n}+\mu_{i+1 / 2}^{n+1 / 2}\right)\left(u_{i+1 / 2}^{n+1 / 2}-u_{i}^{n}\right)=0 \\
\left(c_{i+1}^{n}+c_{i+1 / 2}^{n+1 / 2}\right)\left(\mu_{i+1 / 2}^{n+1 / 2}-\mu_{i+1}^{n}\right)-\left(\mu_{i+1}^{n}+\mu_{i+1 / 2}^{n+1 / 2}\right)\left(u_{i+1 / 2}^{n+1 / 2}-u_{i+1}^{n}\right)=0
\end{array}\right\}
$$

Using an iterative process, the second component of the flux $A p_{i+1 / 2}^{n+1 / 2}$ is computed by solving for $p$ the relationship between pressure and mass of the fluid

$$
\begin{aligned}
\mu_{i+1 / 2}^{n+1 / 2}= & \mu_{\text {Ref }} \\
& +\frac{A}{c^{2}}\left[p-p_{\operatorname{Ref}}+\left(p_{\text {Ref }}^{-1 / \theta}-p^{-1 / \theta}\right) \alpha \rho \eta_{\operatorname{Ref}} p_{\operatorname{Ref}}^{1 / \theta}\right]
\end{aligned}
$$

The treatment of boundary conditions is separately presented for a better understanding. The computing of the local interface fluxes at the left-hand $(i=1 / 2)$ and righthand ( $i=N+1 / 2)$ boundaries is carried out by a standard process. For a prescribed pressure, they are respectively

$$
\begin{aligned}
& u_{1 / 2}^{n+1 / 2}=u_{1}^{n}+\frac{\left(c_{1}^{n}+c_{b}\right)\left(\mu_{b}-\mu_{1}^{n}\right)}{\mu_{b}+\mu_{1}^{n}} \\
& u_{N+1 / 2}^{n+1 / 2}=u_{N}^{n}+\frac{\left(c_{N}^{n}+c_{b}\right)\left(\mu_{N}^{n}-\mu_{b}\right)}{\mu_{b}+\mu_{N}^{n}}
\end{aligned} .
$$

For the case of a prescribed flow discharge, left-hand $(i=1 / 2)$ and right-hand $(i=N+1 / 2)$ boundaries are given by $\left[t_{n}, t_{n+1}\right] \times\left[x_{1-1 / 2}, x_{1+1 / 2}\right]$ for all cells given as

$$
\begin{aligned}
& \mathbf{U}_{i}^{n+1, x}=\mathbf{U}_{i}^{n}+\frac{\Delta t}{\Delta x_{i}}\left(\mathbf{F}_{i-1 / 2}^{n+1 / 2}-\mathbf{F}_{i+1 / 2}^{n+1 / 2}\right) \\
& \mathbf{U}_{i}^{n+1, x}=\left[\begin{array}{c}
\mu_{i}^{n+1, x} \\
Q_{\mathrm{m}, i}^{n+1, x}
\end{array}\right]
\end{aligned}
$$

To incorporate the source term $\mathbf{S}\left(\mathbf{U}_{i}^{n+1, x}\right)$, it is assumed that there are no spatial variations for $\mathbf{U}(x, t)$, and $\mathbf{U}_{i}^{n+1, x}$ is the starting point,

$$
\begin{aligned}
& \mathbf{U}_{i}^{n+1}=\mathbf{U}_{i}^{n+1, x}+\mathbf{S}\left(\mathbf{U}_{i}^{n+1, x}\right) \Delta t \\
& \mathbf{U}_{i}^{n+1}=\left[\begin{array}{l}
\mu_{i}^{n+1} \\
Q_{\mathrm{m}, i}^{n+1}
\end{array}\right]=\left[\begin{array}{c}
\mu_{i}^{n+1, x} \\
\frac{Q_{\mathrm{m}, i}^{n+1, x}+\rho g A}{1+\left|Q_{\mathrm{m}, i}^{n+1, x}\right| f_{\mathrm{D}} \Delta t}
\end{array}\right],
\end{aligned}
$$

where $\mathbf{U}_{i}^{n+1}$ is the final solution at the end of the time step $t=n+1$.

However, there are other high-order schemes used to improve some numerical results. We only applied firstorder Godunov's method (Eqs. 5-13) which is easier to be 


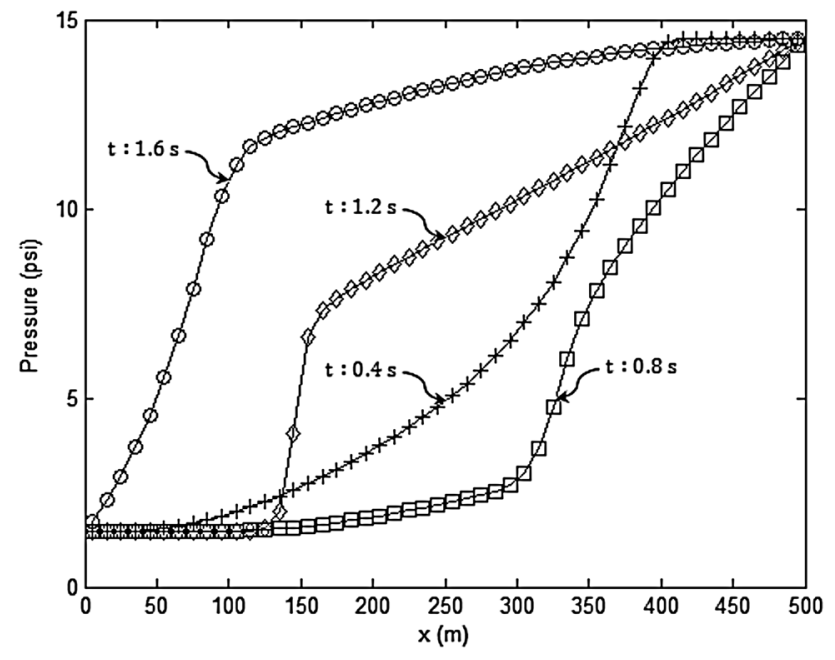

Fig. 2 Numerical solution of two-phase flow in pipe. After Guinot (2003)

implemented and encoded in any programming language (Guinot 2001).

Moreover, there is a similar reported application considering flow in a pressurized pipe (Guinot 2003). It consists of analyzing the dependence between the sound celerity and pressure for two-phase flow in pipes. The physical domain is a circular pipe of $500 \mathrm{~m}$ and $1 \mathrm{~m}^{2}$ of length and cross area, respectively. The working fluid has density of $1000 \mathrm{~kg} / \mathrm{m}^{3}$ and sound celerity of $1000 \mathrm{~m} / \mathrm{s}$. The void fraction is assumed constant at $0.2 \%$. The transient phenomena start from the static fluid at pressure of $14.5 \mathrm{psi}$; then, the pressure at the left-hand boundary is lowered to $1.45 \mathrm{psi}$. It causes a rarefaction wave traveling to the right. When the wave reaches the right-hand boundary, it reflects and propagates to the left along the pipe. Herein, the evolution of the pressure profile has been replicated in order to adequately extend this scheme for oilwell simulations.

The computation of data plotted in Fig. 2 honors the numerical parameters and computation schemes described by Guinot (2003). Logically, this strategy drives to assure consistency, stability of our numerical model, and to optimize the time budget for oil-well simulations.

\section{Dynamic modeling of managed pressure drilling}

In this section, the main engineering contribution of this research is presented. It deals with how the Godunov method is utilized to describe transient hydraulics throughout oil-well drilling, Eqs. 5-13. The well data utilized for modeling are taken from a standard of American Petroleum Institute standards (API RP-13D 2003). Additionally, in order to address more properly engineering concerns, the units used for variables, parameters, and results are in oil-field units.

The fluid "mud" circulation is briefly described as follows: at surface conditions, the mud is pumped down through the drill string and flows to the drill bit; then it circulates back to the surface by the annular space. Regarding the managed pressure drilling, the hydraulics previously stated is perturbed with controlled variations of the choke pressure $p_{\mathrm{ch}}$ at the surface end of the annulus (Table 1).

\section{Initial-boundary conditions}

The initial conditions are related to static or steady oil-well data. On the other hand, the ends of the computational domain define the boundary locations. The left-hand boundary is located at $i=1 / 2$, Fig. 1 . This cell corresponds to the point at surface where the mud is injected down by the stand pipe. The known variable is a constant flow rate of liquid, $280 \mathrm{gpm}$ (Table 1) and the transient pumping pressure $P_{\text {pump }}$ is the unknown data. The righthand boundary is located at $i=N+1 / 2$ and represents the last annular cell where the drilling fluid leaves the oil well. At this point, the data of choke pressure $p_{\text {ch }}$ are consistently modified based on typical field practices. The liquid flow rate is computed at each time step for describing the dynamic behavior of the oil-well hydraulics. The time spacing between right-hand boundaries is related to the overall time spent by the pressure waves for traveling along the entire well.

For the Godunov numerical model to have stability and consistency during computation, we are utilizing the

Table 1 Boundary conditions for dynamic modeling of managed pressure drilling

\begin{tabular}{lllll}
\hline & Symbol & Parameter & Value & Time (s) \\
\hline Left-hand boundary, $i=1 / 2$ & $Q_{\mathrm{L}}$ & Liquid flow rate & $280 \mathrm{gpm}$ & Overall \\
Right-hand boundary, $i=N+1 / 2$ & $p_{\mathrm{ch}, 1}$ & Choke pressure & $100 \mathrm{psi}$ & 7.4 \\
& $p_{\mathrm{ch}, 2}$ & Choke pressure & $200 \mathrm{psi}$ & $50 \mathrm{psi}$ \\
& $p_{\mathrm{ch}, 3}$ & Choke pressure & $0 \mathrm{psi}$ & 22.2 \\
\hline
\end{tabular}


hydrodynamic and numerical parameters presented in Table 2 .

\section{Well geometry}

The physical domain is defined by the well geometry detailed in API RP-13D (2003). The depth and diameter of each section are related to the cell size and are also the data for calculating the cross area $A$, where the mud is circulated (Table 3 ). The area of drill string (Well sections 1 and 2) is circular and its inner diameter is the same as the hydraulic diameter, $D_{\mathrm{h}}$. For the annular space (Well sections 3, 4, and 5), the flow area is delimited by the outer diameter of drill string and the inner diameter of the cemented casing or open hole. In most of cases, the drill bit size defines the open-hole diameter.

Table 2 Hydrodynamic and numerical parameters used for transient modeling

\begin{tabular}{lll}
\hline Symbol & Parameter & Value \\
\hline$c$ & Sound celerity & $1000 \mathrm{~m} / \mathrm{s}$ \\
$f$ & Average friction factor & 0.015 \\
$\theta$ & Coefficient in the perfect gas equation & 1 \\
$\eta_{\text {Ref }}$ & Void fraction at reference pressure & 0 \\
$\rho_{\text {Ref }}$ & Liquid density at reference pressure & $1.49 \mathrm{gr} / \mathrm{cm}^{3}$ \\
$\varepsilon_{\mu}$ & Tolerance criterion on $\mu$ & $1 \mathrm{E}^{-6}$ \\
$\varepsilon_{u}$ & Tolerance criterion on $u$ & $1 \mathrm{E}^{-6}$ \\
$I_{\text {Max }}$ & Limit number of iterations & 100 \\
$N$ & Number of cells in the model & 730 \\
$N_{\mathrm{b}}$ & Cell at annular bottom-hole depth & 365 \\
$t_{\text {Max }}$ & Time length of the simulation & $50 \mathrm{~s}$ \\
$\Delta x$ & Cell size & $10 \mathrm{~m}$ \\
$\Delta t$ & Maximum time step & $0.01 \mathrm{~s}$ \\
\hline
\end{tabular}

\section{Results of transient modeling}

After the description of the well geometry, boundary conditions, hydrodynamic, and numerical parameters, the results of transient pressure and transient flow rate for managed pressure drilling will be discussed and validated with actual well data taken from a standard of American Petroleum Institute (API RP-13D 2003). This recommended practice provides a basic understanding and guidance about drilling fluid rheology and hydraulics, and their application to drilling operations.

In Fig. 3, the well's schematic generated using the data of Table 3 is located in the left figure. The pore pressure profiles (green lines) against depth correspond to the static equivalent densities of $1.0-1.4 \mathrm{gr} / \mathrm{cm}^{3}$; therefore, the working fluid designed has density of $1.49 \mathrm{gr} / \mathrm{cm}^{3}$. These mechanical data are the main constraints for safely drilling the rock formation (Nicolás-López et al. 2012). Therefore, they are the initial conditions and it is named as static well condition, Eq. 3. The transient phenomena (blue lines) start when the pumps are turned on to inject down $280 \mathrm{gpm}$ of "mud." Figure 3 (left-side) shows that at $0.5 \mathrm{~s}$ the pressure wave front reaches $500 \mathrm{~m}$ into the drill string. Next, it travels as follows: $1.5 \mathrm{~s}$ at $1500 \mathrm{~m}$ and $2.5 \mathrm{~s}$ at $2500 \mathrm{~m}$. In these depth stations, the pressure discontinuities decrease from 1332 to 1186 psi, to $1096 \mathrm{psi}$, respectively. This fact is due to the pressure drop as friction is increased along well depth and even the annular space is at static condition. Also, special interest is focused on when the pressure discontinuity reaches the bottom hole, it occurs at $3.7 \mathrm{~s}$ for this flow conditions. In consequence, the total time spent for the pressure wave to travel along the oil well is $7.4 \mathrm{~s}$. At this time, it is considered that the well is in flowing conditions.

Table 3 Geometry of the well sections described by depth and flow areas

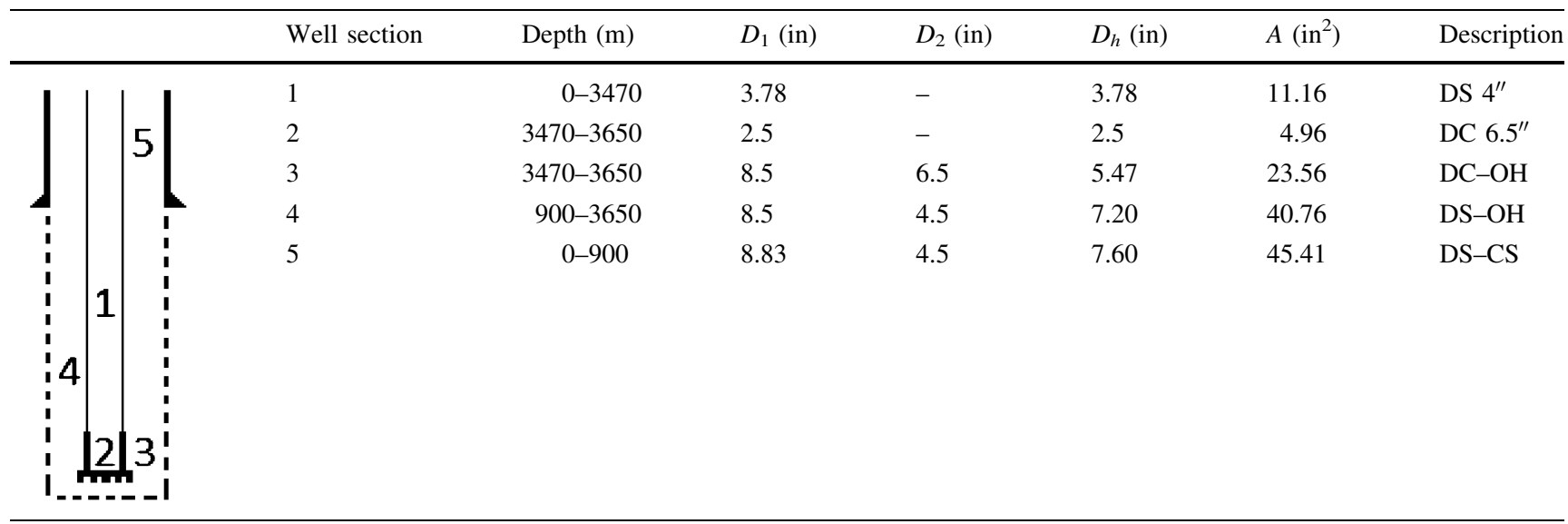

$D S$ drill pipe, $D C$ drill collar, $O H$ open hole, $C S$ cemented casing 


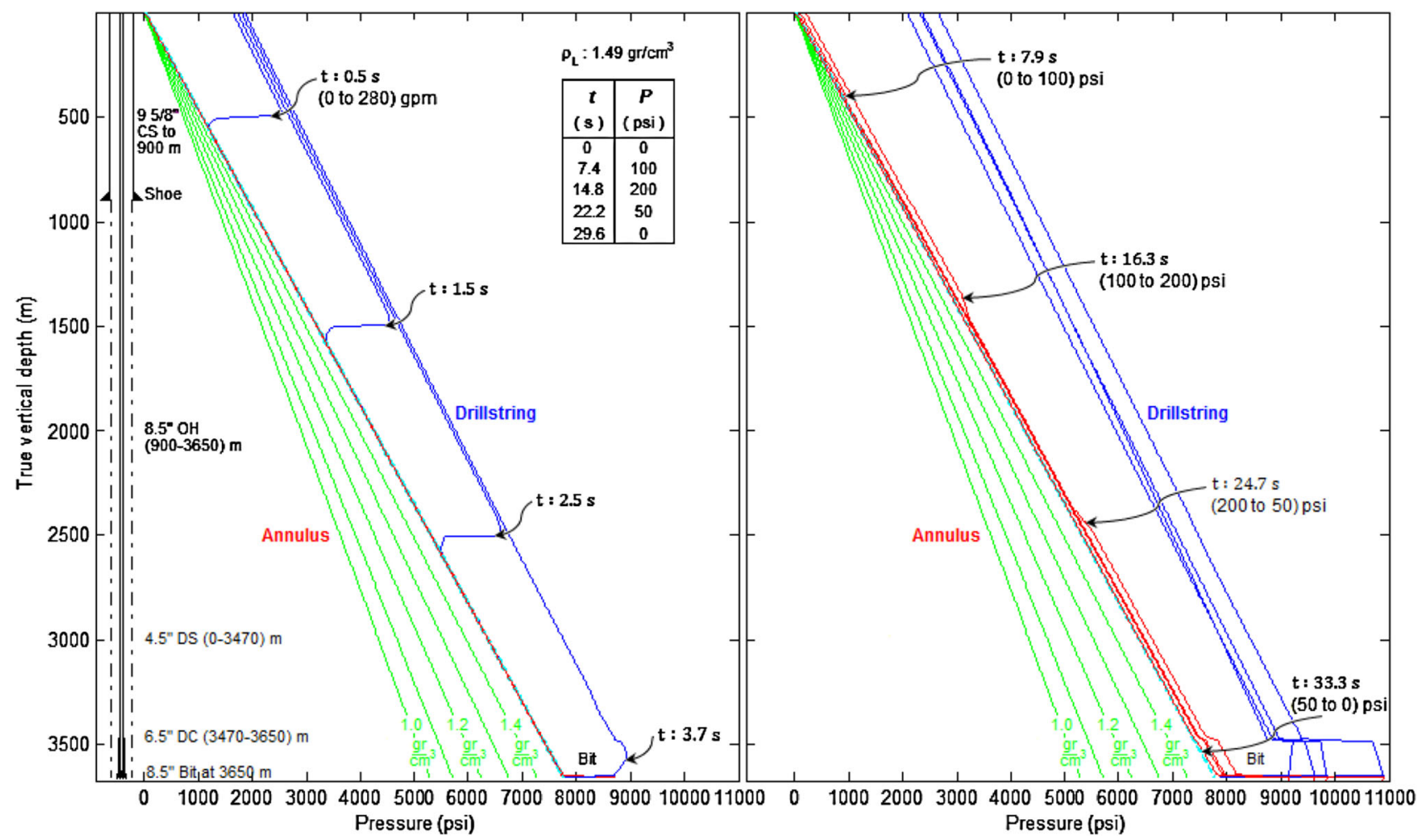

Fig. 3 Onset of fluid circulating and pressure wave traveling through drill string and annulus

In Fig. 3 (right side), the well is flowing and the technique of managed pressure drilling can be analyzed. The blue (drill string) and red (annulus) lines indicate the profiles of transient pressure as a result of controlled changes in the choke pressures (Table 1). For a better visualization of wave traveling, the simulation of pressure profile is plotted for 7.9, 16.3, 24.7, and $33.3 \mathrm{~s}$. The values of corresponding discontinuities are $87,244,-277$, and $-42.6 \mathrm{psi}$, respectively. The minus sign (-) means that the pressure wave front decreases on the flow direction through the annular space.

In Fig. 4, we are summarizing the complete history of the simulation considering $t_{\mathrm{Max}}=50 \mathrm{~s}$. The predicted hydraulics is validated against numerical data obtained from API RP-13D (2003). Once again, the red line indicates annulus and blue line is for drill string. The right-hand boundaries $(i=N+1 / 2$, Table 1) are sketched in the upper section, and the well scheme (Table 3 ) is depicted on the right side. The profiles of transient pressure (upper part) and transient flow rate (lower part) are plotted separately at well depths of surface $(0 \mathrm{~m}), 1500 \mathrm{~m}$ (didactic simulation), and bottom hole $(3650 \mathrm{~m})$.

Bear in mind that for left-hand boundary $(x=0 \mathrm{~m}$ and $i=1 / 2$ ), the liquid flow rate $Q_{L}=280 \mathrm{gpm}$ is the known parameter and the pumping pressure $P_{\text {pump }}$ is the worthy computed parameter for oil-field drilling operations.
Another critical parameter is the flowing bottom-hole pressure BHP ( $x=3650 \mathrm{~m}$ and $i=N_{\mathrm{bh}}$ ), given its variations concerning with the pore pressure described in Fig. 1 . When the pressure choke $P_{\mathrm{ch}}$ is step increased from 0 to $100 \mathrm{psi}$ to $200 \mathrm{psi}$, the pumping pressure is smoothly increased following the next relationship between $t$ and $P_{\text {pump }},(0.1 \mathrm{~s}$ at $1607 \mathrm{psi}),(7.4 \mathrm{~s}$ at $2058 \mathrm{psi}),(14.8 \mathrm{~s}$ at $2286 \mathrm{psi})$. However, when $P_{\mathrm{ch}}$ is stepped down from 200 to $50 \mathrm{psi}$ to $0 \mathrm{psi}$, there are two pressure wave fronts as follows (22.2 s at 2348-2551 psi), (29.6 s at 2772-2585 psi); finally, the calculated data are validated for $50 \mathrm{~s}$ at 1949 psi. This last $P_{\text {pump }}$ is only $7 \%$ different from $P_{\text {pump }}=2097$ psi reported in the API RP-13D (2003).

In the case of bottom-hole pressure BHP $(3650 \mathrm{~m})$, the initial value of 7735 psi corresponds to the hydrostatic head exerted by the mud density of $1.49 \mathrm{gr} / \mathrm{cm}^{3}$. After the transient flow rate has crossed this depth, the BHP is mainly changing as a result of the stepped changes of $P_{\mathrm{ch}}$ (Table 1) and the friction pressure drop in the annulus. The variations are as follows: (7.4 s at $8023 \mathrm{psi}),(14.8 \mathrm{~s}$ at $8111 \mathrm{psi}),(22.2$ at $8453 \mathrm{psi})$, and (29.6 s at 7902$)$. Similar to $P_{\text {pump }}$, we are validating the $\mathrm{BHP}=7895 \mathrm{psi}$ and BHP $+\Delta P_{\mathrm{Bit}}=8993$ psi against the pressures of 7979 and 9005 psi, respectively. They have less than $1 \%$ of difference between computed and reported data (API RP13D 2003). 

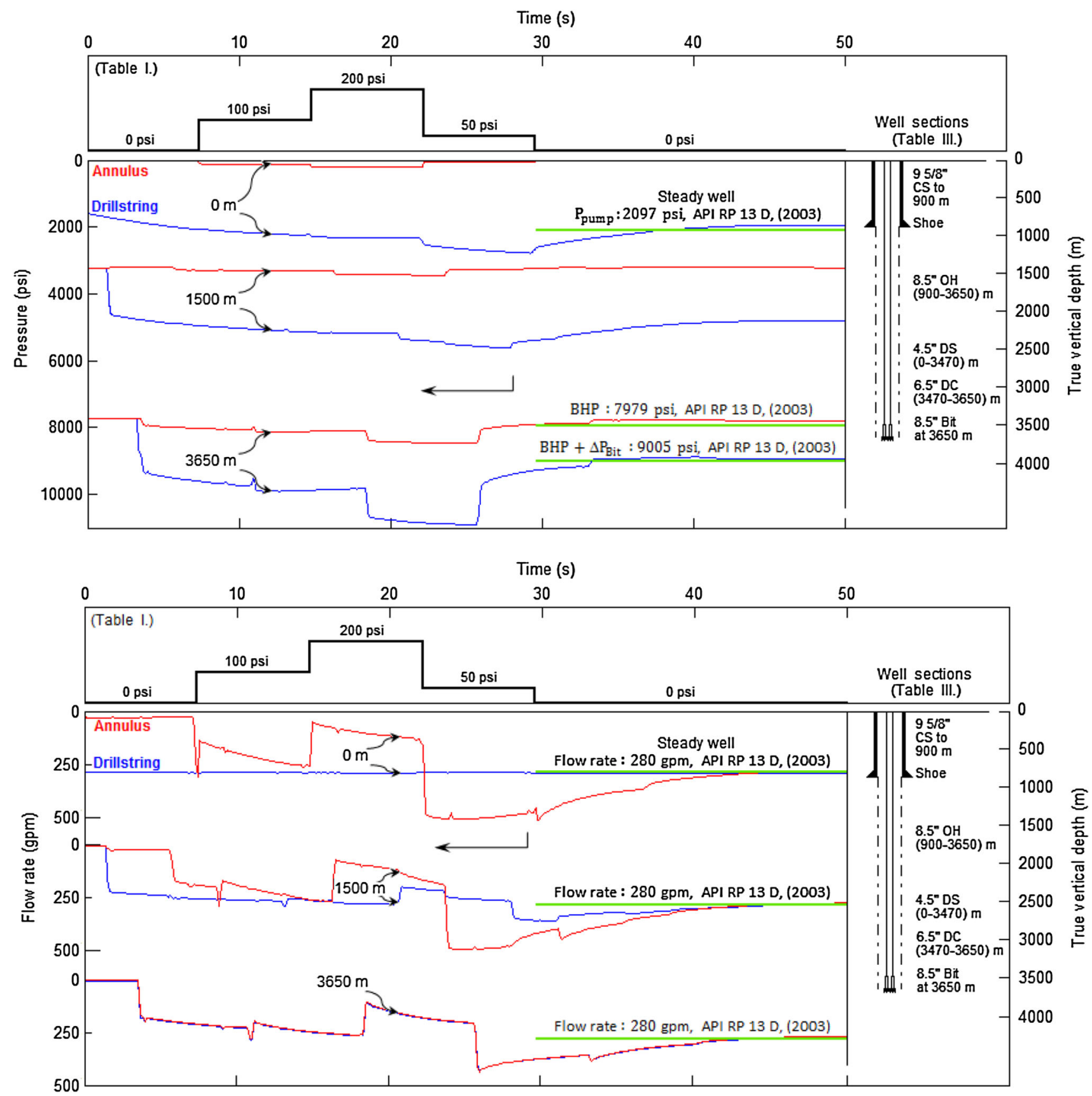

Fig. 4 History of simulation including profiles of transient pressure and transient liquid flow rate

Special attention is focused on the profile of transient flow rate $Q_{\mathrm{L}}$, (lower part, Fig. 4). The left-hand boundary (Eq. 3; Table 1), $Q_{\mathrm{L}}=280$ gpm remains constant for the whole simulation. It is depicted by the blue line at $0 \mathrm{~m}$. However, in order to explain some slugging flow commonly observed at the surface end of the annular space, $Q_{\mathrm{L}}$ at the right boundary $(i=N+1 / 2)$ shall be discussed in detail. First, at $7.4 \mathrm{~s}, Q_{\mathrm{L}}$ is increased from 0 to $283 \mathrm{gpm}$, and it is related to the time spent by the pressure wave traveling along the oil well (Fig. 3). Next, at $14.8 \mathrm{~s}$, there is a sudden decrease from 254.6 to $52 \mathrm{gpm}$ as $P_{\mathrm{ch}}$ changes up to 200 psi. The opposite transient effect is reflected when $P_{\text {ch }}$ decreases to $50 \mathrm{psi}$ at $22.2 \mathrm{~s}$, then $Q_{\mathrm{L}}$ rises from 148 to $498.5 \mathrm{gpm}$. The oil well continues discharging at $29.6 \mathrm{~s}$, and the liquid flow rate slightly increases from 465 to $503 \mathrm{gpm}$. This "up-and-down" behavior of the oil-well hydraulics is supported by the criteria of mass conservation. Moreover, all of these $Q_{\mathrm{L}}$ variations affect directly the 
transient values of friction pressure drop, $\Delta P_{\text {Bit }}$ and $P_{\text {pump. }}$. Finally, the simulating conditions are unaltered until $50 \mathrm{~s}$ to converge to steady state defined by $280 \mathrm{gpm}$ for all well sections.

\section{Conclusions}

The Godunov scheme was applied for modeling transient phenomena during actual oil-well drilling. The pressure wave traveling along the well was described when the managed pressure drilling is utilized. The set of initial and boundary conditions can be consistently established with the oil-well geometry and true operational conditions of the drilling hydraulics. The numerical model based on the finite-volume method was presented and solved using the definition of the Riemann problem for the entire physical domain and time length of simulation. The source term must includes the effects of potential energy together with energy dissipated by the friction mechanism. The simulation was discussed in detail for the most important parameters: choke pressure, pumping pressure, bottom-hole pressure, and circulating flow rate. As computed results are close to reported oil-well data in API RP-13D (2003), the Godunov scheme has high-potential engineering applications for modeling the transient drilling hydraulics, i.e., controlled flow, underbalanced drilling, and foam cementing. Also, implementing high-order Godunov schemes is suggested to improve the quality of computed results, coupling with the heat transfer equations and the models of fluid-rock interaction.

Acknowledgments The authors wish to state their appreciation to Instituto Mexicano del Petróleo for their permission to publish this article.
Open Access This article is distributed under the terms of the Creative Commons Attribution 4.0 International License (http:// creativecommons.org/licenses/by/4.0/), which permits unrestricted use, distribution, and reproduction in any medium, provided you give appropriate credit to the original author(s) and the source, provide a link to the Creative Commons license, and indicate if changes were made.

\section{References}

API RP-13D (2003) Recommended practice on the rheology and hydraulics of oil-well drilling fluids, 4th edn. American Petroleum Institute, Washington

Bilgesu HI, Koperna GJ Jr (1995). The impact of friction factor on the pressure loss prediction in gas pipelines. SPE30996, SPE Eastern Regional Conference and Exhibition, Morgantown

Bousso S, Fuamba M (2013) Numerical simulation of unsteady friction in transient two-phase flow with Godunov method. J Water Resour Prot 5:1048-1058

Brkić D (2011) Review of explicit approximation to the Colebrook relation for flow friction. J Petrol Sci Eng 77(1):34-48

Guinot V (2001) Numerical simulation of two-phase flow in pipes using Godunov method. Int J Numer Meth Eng 50:1169-1189

Guinot V (2003) Godunov-type schemes: an introduction for engineers, Chapter 5. Elsevier, Amsterdam

Kerger F, Archambeau P, Erpicum S, Dewals BJ, Pirotton M (2011) An exact Riemann solver and a Godunov scheme for simulating highly transient mixed flows. J Comput Appl Math 235:2030-2040

Nicolás-López R, Valdiviezo-Mijangos OC, Valle-Molina C (2012) New approach to calculate the mud density for wellbore stability using the asymptotic homogenization theory. Pet Sci Technol 30(12):1239-1249

Toro EF (2009) Riemann solvers and numerical methods for fluid dynamics. Springer, New York 